\title{
Configuración y reconfiguración de una cultura académica en una universidad mexicana
}

Establishing and Re-establishing an Academic Culture in a Mexican University

Configuração e reconfiguração de uma cultura acadêmica em uma universidade mexicana

Florentina Preciado-Cortés* iD orcid.org/0000-0002-9268-7860

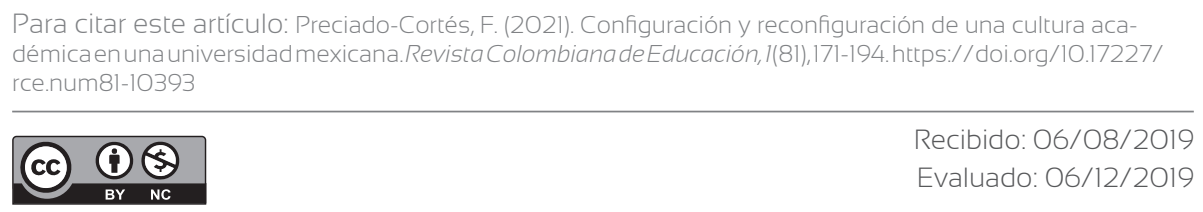




\section{Resumen}

Este artículo de investigación analiza el proceso de gestación y desarrollo de la cultura académica en una universidad mexicana. En sintonía con el tema se llevó a cabo un estudio cualitativo de caso que abordó a los académicos a través de entrevistas sobre sus trayectorias a fin de reconstruir la historia institucional y, por ende, la cultura académica. El análisis muestra cómo el profesorado va construyendo sus procesos y prácticas académicas hasta volverlas propias y naturales del espacio universitario. Sin embargo, la política internacional irrumpe en esa cultura académica propia para reconfigurarla, desplazar el ethos académico e imponer la estandarización de procesos y prácticas de organización universitaria. Sin duda el acercamiento desde la historia oral permite recuperar la memoria colectiva del profesorado para documentar el origen de las prácticas y procesos académicos que forman parte del ambiente privado de las universidades y que han dado vida a la institucionalización.

\section{Palabras clave}

cultura académica; profesores educación superior; política educativa; historia oral

\section{Keywords}

school culture; teachers; higher education; educational policy; oral history

\begin{abstract}
This research article analyzes the emergence and development of an academic culture in a Mexican university. In line with the topic, the authors carried out a qualitative case study. They interviewed academics about their professional careers to reconstruct the institutional history and, therefore, the academic culture. The analysis shows how the faculty members establish their academic processes and practices until they take ownership of them, and they become natural in the university space. However, international politics bursts into that academic culture to re-establish it, displace the academic ethos and impose the standardization of university organization processes and practices. Certainly, the approach from oral history allows recovering the collective memory of teachers to supply documentary evidence of the origin of the practices and academic processes that make-up the private environment of universities and have given life to institutionalization
\end{abstract}

\section{Resumo}

Este artigo de pesquisa analisa o processo de gestação e desenvolvimento da cultura acadêmica em uma universidade mexicana. Em consonância com o tema foi realizado um estudo de caso qualitativo que abordou os acadêmicos por meio de entrevistas sobre suas trajetórias a fim de reconstruir a história institucional e, portanto, a cultura acadêmica. A análise mostra como os professores constroem seus processos e práticas acadêmicas até que se tornem seus e naturais ao espaço universitário. No entanto, a política internacional irrompe nessa cultura acadêmica para reconfigurá-la, deslocar o ethos acadêmico e impor a padronização de processos e práticas de organização universitária. Sem dúvida, a abordagem da história oral possibilita resgatar a memória coletiva do corpo docente para documentar a origem das práticas e processos acadêmicos que fazem parte do ambiente privado das universidades e que têm dado vida à institucionalização.

\section{Palavras-chave}

cultura acadêmica; professores; educação superior; política educacional; história oral 


\section{Introducción}

Desde una perspectiva sociohistórica se hizo la reconstrucción de las formas de trabajo y de organización del profesorado de una universidad mexicana hasta el punto de constituir un esquema simbólico de prácticas, procesos, creencias y valores propios. Este esquema con el paso del tiempo se fue cimentando hasta llegar a convertirse en una estructura que se traduce en una cultura académica. Se entiende que el análisis de la cultura es bastante complejo y, por supuesto, de gran amplitud, por lo que en este artículo se presentan algunos rasgos constitutivos sobre los procesos de organización del trabajo docente, que después se traducen o transforman en lo que hoy se le identifica como cultura académica.

Si bien es cierto que se presenta el análisis particular de una institución también lo es que desde allí es posible generar el debate y el análisis en torno a la experiencia de otras comunidades académicas que en su propio contexto local enfrentan o han enfrentado la reconfiguración de su cultura académica como consecuencia de las políticas internacionales con respecto a la homogeneización del trabajo académico, entre otras cosas.

\section{Referentes teóricos}

Los enfoques utilizados en este artículo provienen de la sociología reflexiva (Bourdieu, 1988, 1995, 1997; Giddens, 1995), la teoría de la acción social (Berger y Luckmann, 1978; Luckmann, 1996) y los estudios psicoanalíticos (Kaës, 1989). Siguiendo esta línea se buscó hacer la reconstrucción de los procesos de gestación y desarrollo de una cultura académica desde la experiencia de los propios protagonistas, a partir del significado que ellos otorgan a sus prácticas, lo cual implica el reconocimiento de los académicos como gestantes de un espacio, pero también como productores de prácticas y de sentido. Al dar a conocer y recuperar las prácticas del profesorado se busca rescatar y reivindicar su papel, al mismo tiempo que se contribuye al conocimiento sobre cómo se configuran estos procesos de cultura, en los que se amalgaman una diversidad de imaginarios, prácticas y saberes que después se traducen en esquemas que norman la vida del trabajo académico.

El tema es complejo y se reconoce que en la cultura académica intervienen múltiples factores que de diversas maneras impactan, pero también se ven impactados por esta reconfiguración académica; entre ellos se encuentran las propuestas de formación académica tanto en licenciatura como en posgrado, las interacciones entre colegas así como con el estudiantado, la percepción de los estudiantes hacia sus profesores en el trabajo presencial y a partir de la incorporación de plataformas tecnológicas; la perspectiva de género que está presente en los discursos internacional,

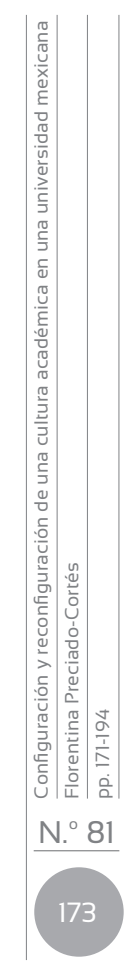


nacional e institucional pero que no termina de concretarse en los espacios y prácticas universitarias, de manera más reciente podemos agregar la internacionalización de la educación superior. Sin embargo, en este escrito no se abordan estos factores, por la propia delimitación del análisis para centrar la atención en el profesorado y la institución, pero también por la extensión permitida.

Así, la intención del artículo es mostrar el proceso de construcción de una cultura académica a partir de la experiencia, la trayectoria y praxis del profesorado a lo largo de casi dos décadas dentro de la estructura institucional de la Universidad de Colima, y de este modo reconocer la particularidad y el origen de las tradiciones académicas.

Como bien lo señalan algunos autores, el impacto de las políticas internacionales ha provocado malestar en el gremio académico (Porter, 2005; Suasnábar, 2001), resistencias (DeVries, 2001; Ordorika, 2004; Porter, 2005), cambios, pero también una adaptación (Porter, 2005; Chavoya, 2006); luego el peso de estas política es tal que condiciona las posibilidades de estrategia de la academia para modificar relaciones de fuerza y de poder. No obstante, como bien lo señala Ordorika (2004), el sujeto amenazado tiene posibilidad de cambiar su condición a partir de su capacidad de análisis y de acción.

Cabe señalar que fue a finales de los años noventa cuando la globalización Ilegó fuertemente a las universidades latinoamericanas (Ibarra, 2001, 2002; DeVries, 2001; Porter, 2003) con duras críticas por las innumerables formas de exclusión que empezaron a darse en las instituciones a partir de una explicación racional sobre las decisiones en función de presupuestos reducidos paulatinamente en las universidades públicas (Glazmán, 2004). En palabras de Ibarra, esta tendencia impulsa a las universidades a hacer uso de "su único activo real, el capital humano de sus académicos, con el propósito de incrementar sus ingresos; tiene que ver con un conjunto de iniciativas y comportamiento económicamente motivados para asegurar la obtención de recursos externos" (2001, p. 45). Desde entonces las universidades se han reconfigurado de manera importante porque los procesos se han homogeneizado, los programas se han sometido a evaluaciones permanentes y los perfiles del profesorado se han estandarizado.

Pero no siempre ha sido así. La memoria de los académicos es única, es la fuente primaria para recuperar el origen de la cultura académica, es a partir de los testimonios que se puede recuperar el pasado, interpretar el presente y vislumbrar el futuro; porque pretender replicar modelos en escenarios distintos, con sujetos y necesidades diferentes, es negar el origen mismo, castigar la diferencia y perder lo original. Averiguar sobre la riqueza de la particularidad de las instituciones conduce a procesos de reflexión mucho más ricos y diversos pues se considera la geografía cultural en la que se manifiestan determinados procesos y sus significados. 
Ahora bien, hablar de cultura frente a la globalización es complicado, sobre todo si se considera que es producto de un grupo en particular. Tal como lo señala Maturana, la cultura

[...] es una red cerrada de conversaciones que define y constituye todo el quehacer de una comunidad humana. Dicho de otra manera, una cultura es un continuo fluir en el lenguaje y las emociones, define y constituye el modo de vida de un grupo humano. (1991, p. 88).

Así, la cultura académica se entiende como un proceso dialéctico entre sujetos que reciben retroalimentación en la praxis e involucra formas de interacción, valores, reglas y patrones de conducta ligados a sentimientos y emociones (Bourdieu, 1988) misma que da sentido al actuar cotidiano. No obstante, como constructo social, la cultura puede mostrar modificaciones o cambios, rupturas o nuevas configuraciones a partir de la interacción de los propios sujetos y las necesidades del grupo.

\section{Esquema metodológico}

Debido a la naturaleza del tema, el estudio se llevó a cabo desde un paradigma alterno denominado cualitativo o interpretativo. ${ }^{1} \mathrm{El}$ acercamiento empírico se dio a través de la historia oral, que Aceves define como "una praxis del conocimiento histórico sobre la realidad social contemporánea" (1996, p. 24) y la investigación documental, con el propósito de conocer y comprender la experiencia humana (véase también Aceves, 1996, 2009; Meyer y Olivera, 1971; Sígala et al., 2017).

Se realizaron entrevistas semiestructuradas enfocadas a doce personas (siete hombres y cinco mujeres). En ellas se exploró la trayectoria laboral y profesional de los participantes, todos ellos académicos provenientes de distintas disciplinas: medicina, biología, psicología, contabilidad, letras, literatura, ciencias políticas, pedagogía y enfermería; los participantes se formaron en esta y en otras instituciones. Un criterio determinante para seleccionarlos fue que tuvieran más de diez años de haberse incorporado a la universidad y que su contratación inicial fuera para labores de docencia.

A grandes rasgos y por cuestión de espacio se puede señalar que en la entrevista se abordaron tres momentos de las trayectorias: 1) el inicio de la carrera académica, cómo y cuándo llegaron a la universidad,

1 Con los datos fenomenológicos obtenidos se realizó el análisis a partir del enfoque de la "teoría fundada, en el que se entremezclan tanto el análisis inductivo como el deductivo para entender la naturaleza de las experiencias, el rol activo de los sujetos en el mundo en que viven, resaltando la importancia de los cambios, los procesos y las acciones, producto de las interrelaciones que se dan desde ciertas condiciones y con ciertos significados (Strauss y Corbin, 2002). 
así como una descripción de la vida universitaria durante las décadas de los ochenta y los noventa; 2) los momentos más significativos en su trayectoria académica, caracterización del gremio académico y las transformaciones en el trabajo académico; 3) Sus expectativas hacia la institución, principales decisiones y cambios institucionales en relación con la carrera académica.

Aunque la institución se fundó en 1940, para el estudio se decidió analizar el desarrollo de la década de los ochenta, ya que históricamente este periodo es un parteaguas para la universidad, simbólicamente es muy importante pues hubo un proceso de refundación enfocado en la redefinición de la organización, las funciones y el rumbo de la universidad. La década de los ochenta y los primeros años de los noventa conforman el periodo que se considera que dio origen a la gestación y el desarrollo de una cultura académica en la que el profesorado tiene plena libertad para proponer, crear y poner en marcha una infinidad de proyectos académicos que germinan de manera exitosa, y que posteriormente le dieron reconocimiento a la institución. Los testimonios señalan de manera reiterativa que fue una etapa de integración de la comunidad, en la que se fortalecieron lazos académicos y de amistad, en la que su participación fue reconocida y apoyada por las autoridades. El discurso oral hace evidente el entusiasmo y la dedicación de los jóvenes profesores por ser parte de una comunidad que valora su trabajo.

Esta experiencia y el sentir de los profesores se recuperó a partir de la voz de los implicados, en el relato de su trayectoria académica y desde el significado que ellos mismos le otorgan a su práctica cotidiana en relación con los pares y con la institución. Si bien es cierto que el estudio de los académicos de esta universidad no se puede generalizar, sí permite conocer y rescatar la experiencia de crear comunidad académica, por lo tanto puede contribuir al conocimiento sobre cómo se configuran estos procesos en los diferentes espacios para poder entender y reconocer la singularidad de las universidades.

Ahora bien, el artículo se estructura de la siguiente manera: en la primera parte se presenta un panorama general de las coordenadas sociopolíticas de la educación superior a finales de los noventa y principios del año 2000, políticas que básicamente se concretan en el esquema de la productividad y competitividad. Posteriormente, se expone la reconstrucción del espacio académico en la Universidad de Colima, en la que se muestra el impacto de los programas encaminados al mejoramiento del profesorado, en la tercera parte se exponen los elementos configurativos de la cultura académica en esta universidad y el choque con la imposición de los esquemas reguladores del trabajo académico. Finalmente se presentan algunas consideraciones sobre lo expuesto. 


\section{Coordenadas sociopolíticas}

Las políticas desempeñan una función muy importante pues coyunturalmente contribuyen a reformar, desde el Estado, el funcionamiento del sistema educativo. Por medio de ella se institucionalizan nuevas pautas de comportamiento entre sus protagonistas, de esta forma se acercan sus acciones al supuesto modelo de competencia perfecta (Coraggio, 1995), basado en la interacción de demandantes y oferentes de servicios educativos que definen qué educación debe ofrecerse, cuáles contenidos, de qué manera, en qué áreas y a qué precio.

Esta nueva concepción de funcionamiento de la sociedad y de la educación transforma de manera importante la relación que el individuo establece con la sociedad y sus instituciones, es decir, el modo en que se construyen y se desestructuran en el mismo proceso dialéctico de su constitución como agentes que producen pero que también lo consumen todo (Giddens, 1998; Juárez, 2000), que habilitan pero que también constriñen.

El impacto de esta perspectiva permea o contamina los procesos productivos y culturales de manera significativa en lo que se refiere a la creación y reproducción de la cultura. La cultura hace referencia a ese elemento de cohesión y de diferenciación social que da sentido a las conductas de los actores sociales a partir de las cuales construyen su propia imagen (Juárez, 2000). Pero sobre todo puede entenderse como el conjunto de ideas, técnicas, creencias y valores que definen a un grupo, las cuales pueden traducirse en los compromisos que asume o comparte un grupo (Becher, 2001).

Cabe mencionar que dentro de este nuevo esquema social la intervención de la dimensión económica aplicada a la política ha tenido consecuencias sintomáticas muy importantes y que se ven reflejadas en el trabajo que se realiza en las instituciones de educación superior. Muestra de ello es la pugna estratificada entre las instituciones para conservar su puesto o aspirar a un nivel mejor, porque quedar en el estrato subterráneo significa tener que luchar para evitar la ignominia y la humillación pública (Neave, 2001). La competencia conduce a la diferenciación, fortalece al fuerte, debilita al débil y redistribuye los lugares en las posiciones medias.

Esta competencia invade todos los niveles y se manifiesta tanto en el exterior (con otras instituciones) como en el interior (entre los departamentos o las facultades); generalmente se vincula con indicadores de calidad, con procedimientos regulares de revisión, control y evaluación; y lo principal, la asignación de recursos queda sujeta a los resultados, es decir, el financiamiento queda ligado y condicionado a la productividad. Con esta lógica se gratifica a las instituciones que muestran capacidad de adaptación; lo contrario se castiga con la reducción de apoyos, cancelación de proyectos, cierre de programas y sobrecarga de trabajo. Lo más grave 
de este escenario es que, al tener jugadores que responden al juego, se vuelve una lógica cotidiana de mayor aceptación y por lo tanto logra que las instituciones promuevan acciones encaminadas hacia la consolidación de esas políticas (Acosta, 2006; Buendía et al., 2019; Clark, 1991; DeVries, 2001; Naidorf, 2005; Porter, 2005; Vargas, 2016; Walker, 2016).

El esquema impuesto se distingue por la competitividad en todos los niveles. Para mantenerse o sobrevivir dentro de esta lógica empresarial se apuesta al fortalecimiento de la capacidad de gestión a partir de la planeación, el desarrollo y la producción de información sobre el rendimiento institucional, lo que permite, hasta cierto punto, disminuir los riesgos y asegurar una mejor productividad laboral con un control mayor de las actividades. Para que esto sea posible, se fortalece el cuerpo de administradores universitarios encargados de verificar el acatamiento institucional a la reglamentación nacional, y de esta manera asegurar un nivel mayor de adaptación al sistema (Galaz y Gil, 2009; Neave, 2001; Padilla et al., 2013; Pedró, 2004; Walker, 2016).

De este modo, el trabajo académico en las universidades mexicanas se ha visto fuertemente impactado por este tipo políticas educativas que cada vez más condicionan la asignación de los recursos al cumplimiento de modelos pensados desde fuera de la lógica académica. En otras palabras, las políticas educativas están determinando de manera sustancial la organización y redefinición del trabajo académico, lo que por supuesto determina fuertemente el desarrollo y futuro de los académicos (Chavoya, 2001; De Vries, 2001).

En este sentido, la organización y redefinición del trabajo académico es una realidad de nuestras instituciones; desde hace casi veinte años con la implementación del Programa de Mejoramiento del Profesorado (Promep), ahora conocido como Programa para el Desarrollo Profesional Docente (Prodep), ${ }^{2}$ se buscó impulsar de manera específica una sólida formación del personal académico, elevando la calidad de los profesores y mejorando la dedicación a las tareas académicas fundamentales: docencia, generación y aplicación del conocimiento, tutoría y gestión (Anuies, 1997). Asimismo, se ha favorecido la integración de los cuerpos académicos, que tienen como objetivo lograr un nivel de consolidación a partir de criterios como que todos los integrantes cuenten con doctorado, participación en redes de intercambio con pares del país o del extranjero, producción académica sólida entre otros (Secretaría de Educación Pública, Promep, 2003).

2 El cambio de nombre se hizo en el 2014. Esta nueva versión del programa "tiene como objetivo contribuir para que los PTC de las instituciones públicas de educación superior alcancen las capacidades para realizar investigación-docencia, se profesionalicen, se articulen y se consoliden en cuerpos académicos" (http://dsa.sep.gob.mx/prodep.html). 
De acuerdo con la agenda estadística de la educación superior, en 1996 solo el $8 \%$ (de una población total de 14 270) de los profesores de tiempo completo (PTC) contaba con el nivel de doctorado, el $27 \%$ con maestría y el $65 \%$ con licenciatura; después de catorce años de implementarse el Promep, el porcentaje de PTC con grado de doctorado era de 33,81\% (de una población total de 30 129); maestría 51,42 \%, licenciatura el 14,45\% y el $0,31 \%$ no reportaba grado (Promep, 2010).

Analizando el desarrollo de la formación académica de los PTC, se puede observar que, sin duda, el perfil ha cambiado ya que el número de doctores creció de manera significativa. Esta situación es positiva en términos de la formación, pero no lo es tanto si se considera el nivel de exigencia y las tensiones que tuvo que enfrentar el profesorado para cumplir con las metas e indicadores institucionales.

Alcanzar estas cifras provocó que el ambiente institucional se transformara de manera radical, sobre todo por cumplir con el perfil deseable Promep que implica docencia, investigación, tutoría y gestión, así como el compromiso de alcanzar el nivel de doctorado. Tal situación ha sido estudiada y muestra como el profesorado enfrentó de manera dolorosa esta situación (Preciado et al., 2008), debido a que había grandes dificultades para responder a la tarea de gestión y de investigación porque en muchos de los casos no se tenía la formación para desarrollar esta última tarea. En general, la exigencia de cumplir de manera equilibrada con las cuatro funciones sí puso en crisis a una parte considerable de los profesores que tenían una larga carrera como docentes pero no como tutores, gestores e investigadores. Esta visión coincide con la de Acosta (2006), quien señala que la cara descarnada de esta iniciativa del Promep deja ver que

[...] aquellas universidades que quisieran ganar el acceso al cielo de los recursos federales extraordinarios deberían registrar sus cuerpos académicos en los formatos, espacios electrónicos y de papel dispuestos para tal efecto por la Subsecretaría de Educación Superior (SES) y por cada universidad (con una pequeña ayuda de la Secretaría de Hacienda y Crédito Público, quien decide desde hace tiempo las reglas, montos y mecanismos de acceso al dinero federal extraordinario). (P. 81).

Esta situación se vivió en muchas instituciones mexicanas con intensa presión por alcanzar los altos indicadores y puso en jaque al profesorado. Por supuesto no es que fuera malo tener niveles altos en formación y productividad académica, pero la manera de lograrlo sí tuvo un impacto considerable, ya que se pasó por alto que todo proceso demanda de ciertas condiciones y tiempo; llegar a la maduración académica requiere dedicación, interés y tiempo. Así es como se ha vivido en las instituciones durante las últimas dos décadas, con la imposición 
de modelos de trabajo académico que homogeneizan prácticas sin considerar las áreas disciplinares ni las condiciones locales, el origen y desarrollo de las instituciones.

Sin duda, fue muy claro y evidente cómo el sistema natural y original de educación de cada institución fue desplazado por un sistema de competencia en el que la mayoría de las decisiones y acciones académicas empezaron a tomarse a partir de motivaciones económicas (Ibarra, 2001). Así, para asegurar la obtención de recursos, las instituciones hicieron y hacen uso "de su único activo real, el capital humano de sus académicos", denominado por Slaughter y Leslie (1997) capitalismo académico.

La consigna de la política académica es contundente:

[...] depurar de entre las multitudes de profesores mal preparados a quienes sí fueran, parecieran o se esforzaran en ser académicos de verdad (discernidos a partir de indicadores importados, adoptados y adaptados de otros lugares $y$, sobre todo, impuestos) e iniciar la renovación de la planta académica sin los lastres y taras que la expansión sin regulación académica, entre 1960 y 1990, había generado. (Galaz y Gil Antón, 2009, p. 6).

Sin miramientos, el profesorado que no cumplía o no estaba dispuesto a cumplir con los indicadores fue desplazado. No se les consultó sobre alternativas o rutas a seguir (Galaz y Gil Antón, 2009), simplemente dejaron de ser valiosos para las instituciones porque su entusiasmo, experiencia y trayectoria no abonaban para la obtención de recursos; enfrentar esta situación con más de veinte años de trabajo es doloroso e ingrato.

A grandes rasgos es lo que ha sucedido en las instituciones de educación superior mexicanas. El seguimiento a las cifras del avance ha sido sistemático y expuesto de manera significativa, aunque también hay trabajos de corte cualitativo que recuperan la experiencia, el sentir y la identidad del profesorado 3 (por ejemplo, Chavoya 2001; Chavoya, 2006; Gil Antón, 1994; Gil Antón, 2004; Porter, 2003 Preciado, 2013; Preciado et al., 2008). Es así como se ha transitado, en palabras de Gil Antón y Galaz "del señor o señora profesora a la del o la académica que en nuestros días piden ser llamados por sus títulos, no por sus nombres" (2009, p. 6).

Durante la última década la carrera académica se ha desarrollado dentro de una nueva estructura; los mecanismos de institucionalización muestran

3 Recomiendo revisar el estado del conocimiento del Consejo Mexicano de Investigación Educativa (Comie) Estudiantes, maestros y académicos en la investigación educativa. Tendencias, aportes y debates 2002-2011, de manera especifica la tercera parte "Los académicos en México: desarrollos recientes, 2002-2011" coordinada por Jesús Francisco Galaz Fontes. 
[...] un proceso sucesivo de consolidación de patrones normativos de modelos de organización y de esquemas reguladores de interacciones e intercambio de valores sociales y culturales, los cuales buscan mantener y preservar la legitimidad de la carrera académica ya constituida, aun cuando tal propósito entrañe la necesaria contradicción con los diversos valores, intereses, orientaciones organizacionales y colectivas, como lo son las políticas educativas emanadas de la tendencias de modernización y globalización actuales. (Viloria y Galaz, 2013, p. 431).

De esta manera, el espacio de intercambio de ideas, de un trato personalizado y entusiasta en la construcción y desarrollo de proyectos y sueños fue perdiendo su encanto hasta convertirse en una estructura fría y rígida que tiene como centro de interés lograr altos indicadores, en muchos de los casos a través del trabajo rutinario.

\section{La reconstrucción del espacio universitario de la Universidad de Colima}

La Universidad de Colima se ubica en el occidente de México, es una pequeña institución pública que tuvo un crecimiento en infraestructura y estudiantil muy significativo a mediados de la década de los ochenta, gracias al cual Ilegaron académicos de diversas disciplinas a desarrollar proyectos importantes, pues la institución representaba un espacio fértil para crecer y adelantar una carrera académica con plena libertad y apoyo económico. Esos proyectos colocaron a la institución como una referencia internacional y nacional en distintos ámbitos, por ejemplo, el de la tecnología, al crear el Sistema Integral Automatizado de Bibliotecas de la Universidad de Colima (Siabuc) ${ }^{4}$ convertirse en el Centro Nacional Editor de Discos Compactos (Cenedic) y formar parte de la Red de cátedras Unesco en México, todo esto desde finales de los noventa y principios del dos mil. En poco tiempo la institución adquirió reconocimiento y fue ampliando su oferta académica; actualmente cuenta con cinco campus con los cuales cubre la demanda estudiantil en todo el estado. Ofrece 66 programas de licenciatura y 37 posgrados. Cuenta con una población estudiantil de 13527 y un personal docente de 1476, de los cuales solo 396 son profesores de tiempo completo (Universidad de Colima, 2018).

A partir de la breve contextualizada de la institución, es posible observar que estudiar la cultura académica implica hacer referencia a una serie de factores políticos, económicos y sociales externos e internos que conforman el escenario de la vida institucional, pero sobre todo buscan

4 En la actualidad 1128 instituciones manejan Siabuc9 en sus centros de información o bibliotecas. 
rescatar al sujeto académico para reconocer su papel en la reproducción y transformación de su propia praxis (Giddens, 1998); en la reelaboración del concepto de sí mismo, como elemento configurado y configurador de la cultura académica.

La institución universitaria es la estructura dentro de la cual el profesorado desarrolla los conocimientos, habilidades y destrezas tanto para su crecimiento profesional como para el impulso de la misma institución. En este sentido, la universidad se entiende como un espacio de conocimiento, de generación de propuestas pero también de intervención. Se estructura a partir de relaciones entre los sujetos participantes del mismo espacio para conformar un campo político-académico, que se explica por la interacción promovida internamente por cada uno de los participantes, caracterizados por tipos de relaciones que la vinculan con los otros en la estructura. El énfasis en la estructura es vital porque es dentro de ella y a partir de ella que la interacción y la función del académico adquieren sentido (Bourdieu, 1995; Giddens, 1998; Kaës, 1989).

Por la consideración de estas dimensiones, se buscó que el acercamiento al profesorado fuera a través de la historia oral, de tal forma que se logró recuperar la experiencia, la memoria, y de este modo, destacar la versión y visión de la experiencia de los actores reconocidos por la propia historia oral, social e institucional (Aceves, 1998). La riqueza de la historia oral es enorme, pues a través de ella se llega a conocer el mundo social de los sujetos, "mediante la tradición oral se preservan y recrean los más preciados elementos de su cultura e historia pasada, y por lo tanto, de su memoria social" (Aceves, 1998, p. 229). De este modo, el acercamiento al profesorado permitió conocer las referencias y vivencias de los protagonistas para recuperar sus procesos de incorporación y desarrollo, así como las prácticas y los tipos de relación que han construido con la institución.

El discurso de los académicos permitió la reconstrucción en paralelo de la memoria colectiva, que se vincula con la historia institucional y sobre la que se apunta lo siguiente: cuando inició el proceso de reconstrucción de la universidad (finales de los setenta), esta se caracterizó como una etapa de desacuerdos entre la población estudiantil y el gobierno del estado, entre otras cosas las demandas de los estudiantes, que pedían un mayor presupuesto y el respeto a los acuerdos previos como el apoyo para el transporte; finalmente el gobierno estatal termina cediendo ante la fuerza del grupo universitario. Por su parte, la política institucional universitaria buscó limar asperezas y disminuir el nivel de conflicto entre el estudiantado. La estrategia para lograrlo fue iniciar con una etapa de conciliación (principios de los ochenta) caracterizada por cubrir las demandas de los estudiantes y de esta forma no dar lugar a nuevos conflictos, es decir limpiar el ambiente para tener un espacio en condiciones de encaminar el nuevo proyecto. 
Para la institución esta nueva etapa representó la posibilidad de realizar un trabajo permanente y sistemático de visibilizar a la universidad dentro del mapa nacional de educación superior pero también dentro del contexto inmediato. De este modo, se emprendió el proceso de refundación con el firme propósito de sus dirigentes de hacer llegar el trabajo de la institución a todo el estado, que la sociedad se percatara de que tenía una universidad y que el trabajo que en ella se realizaba significaba un apoyo para la mejora de las condiciones sociales, económicas, culturales y políticas del estado.

Mientras tanto, en la institución las autoridades universitarias encaminaron sus acciones hacia la eliminación de uno de los rasgos más negativos que había caracterizado a la institución durante la década de los setenta: la informalidad, reflejada en la inexistencia tanto de documentos curriculares como de programas de asignaturas, así como en la constante ausencia de profesores y alumnos, entre otros aspectos. Prácticamente no existía ninguna normatividad para el trabajo, de tal forma que la actividad de profesores, coordinadores y directivos se regía por el entendimiento y compromiso que cada uno de ellos tenía sobre su trabajo.

En tales condiciones resultaba difícil hablar de la existencia de una universidad. Había muchos vacíos en el desarrollo de las actividades académicas, de tal forma que se tuvo que trabajar desde la organización, planificación, operatividad e imagen de la institución, lo que implicaba ir desplazando poco a poco la vieja estructura. En la sustitución y el reacomodo de la nueva estructura se dieron procesos de apertura para nuevo personal, pero también de expulsión, se abrieron nuevos espacios de autoridad y se cerraron cotos de poder; se reestructuró la oferta educativa con nuevas opciones terminales, y se dio paso a la investigación y la difusión cultural como otras funciones de la institución.

Con el nuevo proyecto de universidad (1984), el rector Jorge Humberto Silva Ochoa inauguraba una estructura organizacional con una nueva distribución de funciones, una oferta de carreras profesionales más acorde a las necesidades y requerimientos sociales, así como prácticas de interacción formales entre profesores, alumnos y personal administrativo/ directivo. De este modo se gestaba una nueva generación de académicos (en muchos de los casos bajo tutela de académicos externos que se sumaron al proyecto), pero sobre todo se inició la gestación de un espacio legítimo desde el cual se reconfiguraban el sentido y la estructuración de la institución para convertirse en una estructura con capacidad de aglutinar a un grupo de intelectuales que contribuyeron a legitimar el trabajo de la institución. 


\section{Elementos configurativos de la cultura académica}

Del proceso de análisis de los relatos, documentos e informes institucionales se obtuvo un esquema de los elementos configurativos de la cultura académica en la Universidad de Colima, es decir, la manera en que los académicos han venido trabajando y articulando sus acciones e interacciones dentro y con la institución.

\section{Relaciones de estructura (entre campo académico y la estructura institucional)}

Vinculación dinámica entre las condiciones del contexto y la temporalidad institucional, es decir, el ajuste e interacción que se produce en las relaciones conforme hay cambios y movimientos de ambos lados. Las etapas de este proceso se caracterizan por encuentros, negociaciones, compromisos, fricciones y rupturas; es así como surge la reconfiguración de una nueva forma de trabajo a partir del significado de dichos eventos.

De manera irónica, los académicos coinciden en señalar que el proceso de reestructuración y organización que se presentó a inicios de los ochenta, mismo que permitió el impulso y desarrollo de nuevas carreras, la contratación de nuevos profesores y la apertura de los centros de investigación, fue haciéndose mucho más rígido hasta adquirir un significado distinto, la posibilidad de propuesta y de innovación se fue perdiendo para dar paso a la institucionalización de prácticas, todo como parte de la nueva cultura institucional.

Hubo un gran crecimiento de la institución, un gran fortalecimiento en muchas áreas de despegue, el momento de la explosión fue en los ochenta y yo creo que tienen que ver muchas cosas, una de ellas es la presencia del rector en ese momento que era muy visionario... y sabía exactamente qué quería hacer de la universidad, definitivamente no quería pasar como un rector más, quería que su nombre quedara grabado en la universidad y lo logró... (Académica, 48 años, nivel de maestría).

Como que fue madurando la gente que estuvo metida [en el proceso de refundación], se fueron ubicando [en la estructura], se fueron posesionando y después se transformaron en los que controlaron la universidad e incluso viene como un proceso de control de la universidad sobre los estudiantes, por parte de quienes en un principio habían hecho todo lo contrario. (Académico, 49 años, nivel de maestría). 


\section{Relaciones de fuerza (dominación/subordinación)}

Se enfoca a la gestación de un modo de actuar y de relacionarse dentro de la institución en el que se asume al poder como elemento determinante en la trayectoria académica. Tiene que ver con:

» El desde dónde se estructura el campo académico; cómo se conjugan tareas, prácticas, estrategias de acción, responsabilidades, el reconocimiento de logros, etc., de tal manera que los académicos admiten que muchas decisiones dependen de la autoridad rectoral, es decir, lo académico cuenta, pero no siempre es fundamental. En una entrevista hecha al rector en 1998, describía su función de la siguiente manera:

Me ha tocado interpretar la aspiración de muchos... de dotar al estado de un centro de altos estudios capaz de llenar las expectativas de los que acuden a él para preparase y, por otra parte, responder a los requerimientos profesionales del aparato productivo; a los de difusión de la cultura y extensión de los servicios sociales y educativos para la mayoría de la población... No creo en los individuos providenciales, pero sí en la real posibilidad de concertar voluntades y encauzar la fuerza creadora del conjunto. Esta función tan honrosa me ha tocado durante casi una década. Desempeñarla cabalmente, ha sido mi mayor anhelo. (Cortés, 1988, p. 1).

" La capacidad y el poder de la estructura para generar espacios privilegiados, construir y transmitir una imagen institucional, premiar, sancionar y excluir. Con esta manera de operar mucho más compleja y sofisticada, la estructura institucional hace que los académicos experimenten la presión por cumplir con las cuatro funciones (docencia, investigación, tutoría y gestión) de todo profesor universitario de tiempo completo, pero además, obtener los niveles de formación académica (doctorado) y perfiles deseados (Promep).

Antes de que truene el globo que ocupas, [las autoridades deberían de pensar] quiero que no se truene, pero ¿dónde están los recursos? Seguimos haciendo lo mismo y con mayor responsabilidad, una amiga decía quítenme los títulos y auméntenme la paga, ¿por qué? porque ahora ya no eres licenciado, eres un doctor y además con una serie de estigmas, perteneces al sistema o no, [en eso sí] eres diferente, eres evaluador, director de tesis, [investigador, docente y haces gestión] (Académico, 48 años, nivel de doctorado).

No obtener los perfiles los excluye del reconocimiento institucional, académico y económico, aun contando con mucha experiencia docente. 
[Mi preocupación es] tratar de sacar los grados que pide la institución y con mucha preocupación, pero ya voy a dejar de preocuparme, [porque] qué va a pasar con los sectores que se quedaron o regresaron otra vez al analfabetismo o que no saben qué hacer con tanta información de Internet, nunca vamos a acabar de aprender, pero [en mi trayectoria], hay más satisfacciones que lamentos... [ahora] la escuela cuenta con mejores docentes, mejores instalaciones ipero ay! no hay vinculación con empleos mejor remunerados, con más posibilidades de desarrollo profesional. (Académico, 55 años, nivel de maestría).

» La institucionalización de procesos e intervención de políticas que pre-dirigen. Con la consolidación de las prácticas y la fortaleza de la estructura, el trabajo doméstico con sello original es reemplazado por la homogenización de procesos y actividades, de este modo se avanza hacia la eliminación del sello particular de la cultura académica.

Entonces los trabajos que hacía tenían, servían para los procesos de selección de los aspirantes... recuerdo que en ese tiempo la universidad te daba la libertad de que tú eligieras el proceso de selección, establecieras los criterios, había cierta libertad, ahora ya no... te dicen el promedio de prepa [cuenta] tanto por ciento, el examen de admisión tanto por ciento, el de Ceneval tanto, ya te dan los porcentajes cerrados. Todo lo demás que yo hacía ya no cuenta, lo puedes hacer de manera extra... entonces dejé de hacer prácticamente lo que hacía. (Académico, 49 años, nivel de maestría).

Sin embargo, quienes promueven estas políticas y formas de trabajo están convencidos de que es lo mejor:

Los retos ahora son de una dimensión mayor, tenemos que aprender a trabajar en equipo, tenemos que aprender a tomar decisiones de manera colegiada, tenemos que aprender a mirar el futuro de una manera mucho más ambiciosa... el asunto aquí es que la institución tiene la firme convicción de que debe mejorar, esa es una decisión institucional, es decir de los más altos directivos. (Académico, 52 años, nivel de maestría).

En este sentido, las formas impuestas a las universidades públicas han "implicado una reforma 'suave' a las prácticas autonómicas de la universidad, en las que se ha introducido una sobre-regulación del trabajo académico y administrativo de las organizaciones y del personal académico" (Acosta, 2006, p. 83). Pero el impacto mayor de esta nueva forma de intervencionismo tiene que ver con la manera en que los recursos federales moldean o inducen cambios en los comportamientos de los académicos universitarios (Acosta, 2006). 


\section{Relaciones de sentido (existencial)}

Se trata de las representaciones que los sujetos hacen sobre sí mismos, los imaginarios y el sentido de su permanencia en la institución. Se da una identificación personal y colectiva con la institución; al pertenecer a ella, los académicos tienen una serie de expectativas, pero también enfrentan la desilusión o frustración por deseos no cumplidos, así como por la ruptura de promesas.

De repente siento un poquito de nostalgia, de reclamo, después de quince años de trabajo... sí siento un poquito como de ¡hay Dios! Vale la pena de que [sic] me dedique totalmente al trabajo... yo digo caramba sí me desvelo, sí estoy dando lo mejor de mí para mejorar mi trabajo, merezco un poco más de pago y de reconocimiento, [hasta hace poco] yo estaba endiosada con mi trabajo. (Académica, 45 años, nivel de maestría).

Los académicos sienten que ya no son tan importantes o fundamentales en los proyectos institucionales; como si toda su trayectoria y experiencia quedara anulada ante las nuevas exigencias de la institución, que prácticamente están encaminadas hacia el reconocimiento de niveles y perfiles académicos.

No tengo perfil porque me falta el grado, es uno de los requisitos... los de afuera como que quieren poner el pie en la cabeza, a ese nivel de menosprecio, cuando se supone que quien ha hecho esto somos nosotros prácticamente, ahora llegan y ya está el plato servido y cuando nosotros empezamos era picar piedrita, así nos tocó trabajar con piedras y de ese picar piedras pues es producto esto, lo que tenemos hoy en día. (Académica, 43 años, nivel maestría).

Algunos de ellos sienten que su experiencia es poco valorada, por lo que, en su opinión, la relación con la institución se ha vuelto más fría y distante.

Ese es otro problema de la universidad, que se [le] ha ido, de que no se fija en el perfil, entonces hace que el trabajador dedique más esfuerzo o que de plano diga no le entro porque no tengo el perfil, pero con honestidad, responsabilidad, dedicación yo saque el trabajo, pero también deje los pocos pelos que me quedaban. (Académico, 56 años, nivel de especialidad).

Este profesorado que se incorporó en los ochenta deja ver en su discurso una estrecha relación con la universidad. Aunque hoy esta es una estructura dura y fría, el vínculo emocional con su alma mater se hace evidente en la emoción, el cariño y al mismo tiempo la nostalgia del profesorado al relatar los hechos. De igual modo, hay una manifestación 
de reclamo al sentir el derecho y la necesidad de ser reconocidos como los pioneros, los forjadores, los creadores de lo que hoy es la universidad. Dicho proceso de creación se vincula con el nacimiento, el dar vida, en este caso, con la construcción de un nuevo espacio de desarrollo y de formación académica que el profesorado siente como suyo pues con su participación contribuyó al progreso de la universidad.

Cabe señalar que la percepción cambia si se hace desde la posición del profesorado o de alguien que ha experimentado estar en un mando medio o cargo directivo.

Me siento muy parte de la institución, yo creo que los logros de la institución de alguna manera los siento como míos y creo que haber dejado la institución habría sido un error... afortunadamente esos momentos pasaron y creo que es con la institución que me siento comprometida y donde todavía tendré mucho que hacer. (Académica, 48 años, nivel maestría).

Los testimonios muestran cómo a partir de la refundación inició una nueva etapa para la institución. El éxito de dicho proyecto se debe a que fue concebido y sostenido por factores organizadores inconscientes compartidos por el profesorado de la universidad, en los que se encuentran aprehendidos deseos que la institución permite realizar, esto es lo que posibilita crear el sentido de la universidad, transmitirlo (Fernández, 1994; Kaës, 1996) y mantenerlo a lo largo del tiempo.

Es a partir de la conjunción de estos tres tipos de relaciones que se constituye la cultura académica. El profesorado en sus testimonios hace referencia a formas de interacción iniciales que fueron construyendo un espacio académico y laboral a partir del cual su participación adquiere sentido, sin dejar de reconocer que este espacio está permeado por múltiples manifestaciones de autoridad (Bourdieu, 1988), que no se exponen de manera explícita pero que están detrás de toda acción. Entonces, es a partir de todo este entramado de interacciones que se ha logrado la reproducción de la estructura de manera dual, tanto personal como institucional.

\section{Consideraciones finales}

El estudio sobre las universidades y sus académicos muestra la amplia diversidad y complejidad en las formas de organización de las comunidades académicas mexicanas (Acosta, 2006; Gil Antón et al., 2001); de ahí la existencia de tradiciones, creencias, prácticas y hábitos, lo cual puede ser visto como fortaleza para su continuidad y crecimiento. Es decir, es importante reconocer las diferencias como fuente de riqueza y no solo intentar borrarlas para caminar hacia modelos de estandarización de realidades y perspectivas; como bien lo señala Gil Antón "el dinero es poderoso, pero 
no basta, ni de lejos, para construir una tradición académica" (2006, p. 95). Entonces, ipor qué apostar por la creación de instituciones duras y frías, por qué terminar con lo original, con el trabajo artesanal que impregna un sello de unicidad, de estética, de creación que puede ser insumo fuertemente valioso en la formación universitaria?

Ahora bien, es sumamente valioso buscar diferentes tipos de acercamiento cualitativo a la experiencia del profesorado universitario. En este caso, la historia oral permitió reconocer que lo que se "comunica en el acontecimiento del habla no es la experiencia del hablante como ésta fue experimentada, sino su sentido. La experiencia vivida permanece en forma privada, pero su significación, su sentido, se hace público a través del discurso" (Ricoeur, 1995, p. 10).

Así, el acercamiento a esta institución muestra cómo en la conformación de la cultura académica la articulación de ciertos elementos es detonante. Sin duda, la visión de la autoridad como propiedad estructural permite explicar el funcionamiento de la universidad con acciones encaminadas a modificar, proponer o determinar prioridades de trabajo. De manera paralela, la movilidad en las trayectorias académicas se explica a partir de relaciones de autoridad que legitiman posiciones; en este sentido, las trayectorias muestran la articulación entre los procesos de configuración institucional y la movilidad académica; todo ello forma parte de la cultura académica que es internalizada por el sujeto, de tal forma que rige, da sentido y seguridad a su actividad.

Esta situación ha cambiado en los últimos años, pues ahora son las políticas educativas internacionales las que han intervenido en la vida privada de las universidades, que se conceptualizan como empresas, por lo que se fortalece el fenómeno de sobre-burocratización de la vida académica universitaria, se reduce la libertad para la toma de decisiones y se recrudece la desconfianza (Acosta, 2006), pero también se erosiona de manera importante la cultura académica de cada institución. La homogeneización de prácticas estandariza comportamientos aniquilando el sello personal y creativo del ethos académico.

Pero incluso, se debe reconocer que el poder externo de las políticas se ha infiltrado de manera incisiva en las universidades; para el caso particular de los PTC

[...] el resultado es paradójico: los académicos se vuelven sus propios gerentes, en que tienen que acumular y mostrar evidencias de su propio trabajo, a través de sus propias acciones. Los académicos de tiempo completo han tenido que desarrollar a lo largo de los últimos años habilidades de gestión adicionales a las tradicionales destrezas de docencia, investigación y difusión de sus procesos y productos académicos. (Acosta, 2006, p. 89). 
Lo estudiado muestra la dinamicidad en las prácticas y en los procesos, razón por la cual es posible la regeneración de la actividad de los sujetos, de la estructura, de la identidad, de la cultura, de las relaciones de sentido. Todas estas razones justifican la importancia de hacer un acercamiento al interior de las instituciones. Admitir esta posibilidad de regeneración obliga al investigador a rescatar la historicidad de las prácticas y los procesos en el ámbito universitario para mostrar cómo se gesta y desarrolla una cultura académica que se objetiva en prácticas y procesos.

De igual forma, es importante mencionar que en esta reconfiguración se ha ganado en la sistematización del trabajo, en la planeación educativa pero también ha traído algunos efectos perversos en el profesorado, como la simulación del trabajo, tomar distancia con relación a la institución, así como apatía hacia las reuniones. Por paradójico que resulte, se consume tanto tiempo en planeaciones, metas y objetivos que lo que se atiende al final son las clases y al alumnado.

Finalmente, en cuanto al grupo académico, cabe señalar que en esa vuelta al pasado para traer a la memoria la trayectoria académica con recuerdos como la incorporación a la institución, los logros, los sueños, las expectativas, los cuestionamientos y la incertidumbre es donde se logra poner en evidencia el proceso de retorno del sujeto que le permite tanto a él como al investigador hacer visible ese proceso de rehacer los pasos para resignificarse en un espacio que ha cambiado, que ha sido redefinido y que lo coloca como una identidad en transición. Es precisamente la nueva identidad académica la que debe abordarse en futuros trabajos porque, sin duda, estamos ante una nueva figura y generación de académicos, con formas de trabajo fuertemente determinadas por cuestiones político-económicas. De igual forma, es importante introducir la perspectiva de género en el gremio académico para visibilizar el impacto de estas políticas en las mujeres que desean hacer una carrera académica, y que a pesar del discurso, siguen enfrentando la disyuntiva entre una vida académica o personal.

\section{Referencias}

Aceves, J. (1996). Caminos y geometría de la historia oral reciente en México. En C. Velasco (coord.), Historia y testimonios orales (pp. 23-55). INAH.

Aceves, J. (1998). La historia oral y de vida: del recurso técnico a la experiencia de investigación. En J. Galindo Cáceres (coord.), Técnicas de investigación en sociedad, cultura y comunicación (pp. 207-276). Pearson.

Acosta, A. (2006). Señales cruzadas: una interpretación sobre las políticas de formación de cuerpos académicos en México. Revista de la Educación Superior, 35(139), 81-92. 
Anuies (1997). Programa de mejoramiento del profesorado de las Instituciones de Educación Superior. Revista de la Educación Superior, $101,1-42$.

Becher, T. (2001). Tribus y territorios académicos: la indagación intelectual y las culturas de las disciplinas. Gedisa.

Berger, P. y Luckman, T. (1978). La sociedad como realidad subjetiva. La construcción social de la realidad. Buenos Aires: Amorrortu.

Bourdieu, P. (1988). Homo academicus. Stanford University Press.

Bourdieu, P. y Wacquant, L. J. (1995). Respuestas: por una antropología reflexiva. Grijalbo.

Bourdieu, P. (1997). Capital cultural, escuela y espacio social. Siglo xxı.

Buendía, E. A., Acosta, O. A., Gil, A., A. (2019). En busca de un rostro (in) visible, pero siempre presentes. Revista Mexicana de Investigación Educativa, 24, 80, 15-41. https://www.comie.org.mx/revista/v2018/ rmie/index.php/nrmie/article/view/1236.

Cortés, E. (1988): "Silva Ochoa: no busco la gubernatura". En Cuatro entrevistas a un rector, UCOL, Colima.

Chavoya, M. L. (2001). Organización del trabajo y culturas académicas: estudio de dos grupos de investigadores de la Universidad de Guadalajara. Revista Mexicana de Investigación Educativa, 6, 111. http:// www.redalyc.org/pdf/140/14001106.pdf.

Chavoya, M. L. (2006). Vida académica en las universidades mexicanas: los efectos del trabajo múltiple. En M. L. Chavoya Peña, El trabajo académico en la encrucijada de las políticas. Estudios de caso en la Universidad de Guadalajara. (pp. 129-144). México: Universidad de Guadalajara.

Clark, B. (1991). El sistema de educación superior. Una visión comparativa de la educación superior. Nueva imagen.

Coraggio, J. L. y Torres, R. M. (1995). La educación según el Banco Mundial. Un análisis de sus respuestas y metas. Miño y Dávila Editores-unam.

De Vries, W. (2001). El exorcismo de ángeles y demonios. Los efectos de las políticas públicas sobre el trabajo académico. Universidad Autónoma de Aguascalientes.

Fernández, L. (1994). Instituciones educativas. Dinámicas institucionales en situaciones críticas. Buenos Aires: Paidós.

Galaz F., J. F. y Gil Antón, M. (2009). La profesión académica en México: un oficio en proceso de reconfiguración. Revista Electrónica de Investigación Educativa, 11(2), 1-31.

Giddens, A. (1995). La constitución de la sociedad: bases para la teoría de la estructuración. Buenos Aires: Amorrortu.

Gil Antón, M. (1994). Los rasgos de la diversidad, un estudio sobre los académicos mexicanos. UAM-Azcapotzalco. 
Gil Antón, M. (2004). Amor de ciudad grande: una visión general del espacio para el trabajo académico en México. En El ocaso del gurú. La profesión académica en el tercer mundo (pp. 83-114). México: UAM.

Glazmán, R. (2004). La universidad de papel. En Revista de la Educación Superior, Vol. XXXIII(1), No. 129. s/p http://publicaciones.anuies.mx/ acervo/revsup/129/04.html\#r

Ibarra, E. (2001). La universidad en México hoy: gubernamentalidad y modernización. UNAM.

Ibarra, E. (2002). La "nueva universidad" en México: tranformaciones reicentes y perspectivas. Revista Mexicana de Investigacióm Educativa, 7 (14), pp. 75-105.

Juárez, M. y Comboni, S. (2000). Globalización, educación y cultura. Un reto para América Latina. Universidad Autónoma Metropolitana.

Luckmann, T. (1996)). Teoría de la acción social. España: Paidós.

Maturana, H. R. (1991). El sentido de lo humano. Ediciones Granica.

Meyer, E. y Olivera de Bonfil, A. (1971). La historia oral. Origen, metodología, desarrollo y perspectivas. Historia Mexicana 21(2), 372-387.

Naidorf, J. (2005). Los cambios en la cultura académica a partir de los procesos de vinculación universidad-empresa en las universidades públicas (tesis posgrado), Universidad de Buenos Aires, Argentina.

Neave, G. (2001). Educación superior: historia y política: estudios comparativos sobre la universidad contemporánea. Gedisa.

Ordorika, I. (Coord.) (2004). La academia en jaque. Perspectivas políticas sobre la evaluación de la educación superior en México. CRIM/UNAM/ Porrúa.

Padilla, L., Jiménez, L. y Ramírez, M. (2013). La satisfacción laboral en el personal académico y su relación con la intención de abandonar la profesión. Perfiles Educativos, 25(141), 8-25. http://www.scielo.org. $\mathrm{mx} /$ scielo.php?script=sci_arttext\&pid=S0185-26982013000300002.

Pedró, F. (2004). Fauna académica. La profesión docente en las universidades europeas. Editorial voc.

Porter, L. (2003). La universidad de papel. Ensayos sobre la educación superior en México. CEIICH/UNAM.

Porter, L. (2005). La universidad de las mil y una noches. Education Policy Analysis Archives/Archivos Analíticos de Políticas Educativas, 13, 1-8.

Preciado, F. (2013). Cultura e institucionalización del trabajo académico en la Universidad de Colima. Universidad de Colima.

Preciado, F., Gómez, A. y Kral, K. (2008). Ser y quehacer docente en la última década: un estudio cualitativo del impacto de las políticas de formación del profesorado. Revista de Investigación Educativa, 13(39), 1139-1163. 
Ricoeur, P. (1995). Teoría de la interpretación. Discurso y excedente de sentido, Siglo XXI, México.

Secretaría de Educación Pública. (2012). Programa de Mejoramiento del Profesorado-Promep. Informe ejecutivo [en línea]. http://dsa.sep. gob.mx/pdfs/Informe\%20Ejecutivo\%20Promep.pdf

Sígala, M., Pérez, F., Hernández, G. (2017). El método de historia oral para la reconstrucción de una institución educativa. En Memoria Congreso Nacional de Investigación Edcativa (Comie). http://www. comie.org.mx/congreso/memoriaelectronica/v14/doc/1063.pdf.

Slaughter, S. y Leslie, L. (1997). Academic capitalism: Politics, policies, and the entrepreneurial university. John Hopkins.

Strauss, L. y Corbin, J. (2002). Bases de la investigación cualitativa. Técnicas y procedimientos para desarrollar la teoría fundada. Medellin: Editorial Univerisdad de Antioquía.

Suasnábar, C. (2001). Resistencia, cambio y adaptación en las universidades argentinas: problemas conceptuales y tendencias emergentes en el Gobierno y la gestión académica. Revista Brasileira de Educacao, 17, 50-63.

Universidad de Colima (2018). Numeralia 2018. Recuperado de http:// cenedic.ucol.mx/content/mtransparencia/1025_numeralia2018.pdf.

Universidad de Colima (2019). Siabuc. http://siabuc.ucol.mx/.

Vargas A., Esther J. (2016). La idea de universidad en vilo. Gestión de calidad, capitalismo cognitivo y autonomía. Revista Colombiana de Educación, 72, 139-157.

Viloria, E. y Galaz, F. (2013). Carrera académica. En C. L. Saucedo, Ramos, C. Guzmán Gómez, E. Sandoval Flores y J. F. Galaz Fontes (coord. general). Estudiantes, maestros y académicos en la investigación educativa. Tendencias, aportes y debates 2002-2011 (pp. 427-438). ANUIES, Colección Estados del Conocimiento.

Walker, V. (2016). El trabajo docente en la universidad: condiciones, dimensiones y tensiones. Perfiles Educativos, 28(153), 105-119. 